\title{
Mental workload evaluation of pilots using pupil dilation
}

\begin{abstract}
This study aims to propose a convenient method for evaluating human stress during high cognitive workload in hermetic space via monitoring changes in the eye pupil dilation. An experiment was carried out to establish the correlation between the changes in eye pupil dilation while operating human-machine system and both the pilot's stress level and National Aeronautics and Space Administration Task Load Index (NASA-TLX). The experimental results indicate that detection accuracy of the flight operation task notably decreased and reaction time was prolonged when there was increased mental workload. At the same time, standard deviation of the recorded pupil dilation percentage significantly decreased. This highlights that the differences in eye pupil dilation can be used as reflective measure for mental workload level and performance assessment.
\end{abstract}

Keyword: Pupil dilation; Mental workload; Pilot performance; Human-machine system 